\title{
Atomistic processes during nanoindentation of amorphous silicon carbide
}

\author{
Izabela Szlufarska ${ }^{a}$ \\ Department of Materials Science and Engineering, University of Wisconsin, Madison, \\ Wisconsin 53706-1595 \\ Rajiv K. Kalia, Aiichiro Nakano, and Priya Vashishta \\ Collaboratory for Advanced Computing and Simulations, Department of Materials Science, Department of \\ Physics, and Department of Computer Science, University of Southern California, Los Angeles, \\ California 90089-0242
}

(Received 7 September 2004; accepted 10 November 2004; published online 5 January 2005)

\begin{abstract}
Atomistic mechanisms of nanoindentation of $a$-SiC have been studied by molecular dynamics simulations. The load displacement curve exhibits a series of load drops, reflecting the short-range topological order similar to crystalline $3 \mathrm{C}-\mathrm{SiC}$. In contrast to $3 \mathrm{C}-\mathrm{SiC}$, the load drops are irregularly spaced and less pronounced. The damage is spatially more extended than in $3 \mathrm{C}-\mathrm{SiC}$, and it exhibits long-range oscillations consistent with the indenter size. Hardness is $\sim 60 \%$ lower than in $3 \mathrm{C}-\mathrm{SiC}$ and is in agreement with experiment. The onset of plastic deformation occurs at depth $\sim 75 \%$ lower than in 3C-SiC. (C) 2005 American Institute of Physics. [DOI: 10.1063/1.1849843]
\end{abstract}

Nanoindentation is widely used to study mechanical properties of materials at the nanometer scale. ${ }^{1-4}$ In crystalline solids, many experimental ${ }^{5}$ and theoretical ${ }^{6,7}$ studies show that the nanoindentation load-displacement $(P-h)$ curve is correlated with the nucleation of subsurface defects in crystalline solids. For example, a molecular dynamics (MD) simulation has shown ${ }^{8}$ that nucleation and coalescence of dislocations under an indenter lead to amorphization.

Amorphous materials lack a long-range order of topological network and hence there is no clear notion of dislocations. For this reason, understanding atomistic processes during nanoindentation in amorphous materials presents a great challenge. MD simulations provide trajectories of all the atoms and are expected to shed light on the question of deformations in amorphous materials. The short- and medium-range topology of $a-\mathrm{SiC}$ has been studied by MD simulations.

In this Letter, we study nanoindentation-induced deformations of amorphous silicon carbide $(a-\mathrm{SiC})$ by means of $\mathrm{MD}$. The maximum indentation pressure of $30 \mathrm{GPa}$ is consistent with experimental values of hardness for $a-\mathrm{SiC}$ (Ref. 10 ) and is only $\sim 40 \%$ of that in a zinc-blende crystalline $3 \mathrm{C}-\mathrm{SiC}{ }^{8}$ The indentation damage is spatially less localized in comparison with $3 \mathrm{C}-\mathrm{SiC}$. The onset of plasticity occurs at a depth only $25 \%$ of that in $3 \mathrm{C}-\mathrm{SiC}$. The $P-h$ curve in $a$ $-\mathrm{SiC}$ exhibits a series of load drops in the plastic regime. The magnitudes of load drops are less than in $3 \mathrm{C}-\mathrm{SiC}$ due to less accumulated pressure under the indenter. The load drops are related to changes in structural correlations, which are analyzed through local rearrangements of atoms, local pressure and shear stress distribution, and bond-angle distribution.

The interatomic potential used here consists of two- and three-body terms, which include steric repulsion, charge transfer, electronic polarizability, van der Waals interaction, and covalent bonding effects. The calculated lattice constant, melting temperature, elastic constants, and cohesive energy are in good agreement with experiments. ${ }^{11}$ This potential has been used also to predict a new mechanism for a reversible zinc-blende-to-rocksalt transformation of $\mathrm{SiC}$ under

\footnotetext{
${ }^{a)}$ Electronic mail: izabela@engr.wisc.edu
}

pressure, ${ }^{12}$ which was later confirmed by first-principles calculations. $^{13}$

We start from a perfect zinc-blende bulk $(N$ $=1048320$ atoms) at the experimental $\mathrm{SiC}$ density $\left(3.1275 \mathrm{~g} / \mathrm{cm}^{3}\right)$ and dimensions $320.6 \times 313.9 \times 107.9 \AA^{3}$ in the $x, y$, and $z$ directions, respectively. To create a relaxed $a$ -SiC, we use the melt-quench method with a slow annealing schedule. ${ }^{9}$ The density of the final $a$-SiC substrate is $10 \%$ lower than that of $3 \mathrm{C}-\mathrm{SiC}$, consistent with the experimental observation of the density reduction. ${ }^{14}$

The resulting $a-\mathrm{SiC}$ has been characterized by calculating various structural correlation functions. ${ }^{9}$ The first peak in the $\mathrm{Si}-\mathrm{C}$ pair distribution function $g_{\mathrm{SiC}}(r)$ occurs at $r$ $=1.89 \AA$, i.e., the bond length is the same as in the crystalline $3 \mathrm{C}-\mathrm{SiC}$ structure. The coordination number is found to be 3.76, which is lower than the value 4.0 in crystal. We have not found homonuclear bonds in our sample, and hence it has a high chemical order. The presence of diamond or graphite structures is also insignificant.

Before introducing an indenter, we remove the periodic boundary conditions in the $z$ (i.e., indentation) direction. The indenter is rigid, and it has a flat square base of dimensions $30.8 \times 30.5 \AA^{2}$ and a height of $\sim 60 \AA$. This geometry of the indenter has been used to study the subsurface atomic structure in metals ( $\mathrm{Al}, \mathrm{Au}, \mathrm{Ni}$ ) (Ref. 15 ) and $3 \mathrm{C}-\mathrm{SiC},{ }^{8}$ when plastic activity is of interest. The interaction between the indenter and the substrate is purely repulsive. The indenter is inserted up to $\sim 10 \%$ of the substrate thickness at the rate of $0.9 \mathrm{~m} / \mathrm{s}$ (each $0.5 \AA$ depth increment is followed by a holding phase of $\sim 53 \mathrm{ps}$ to allow for the decay of transient
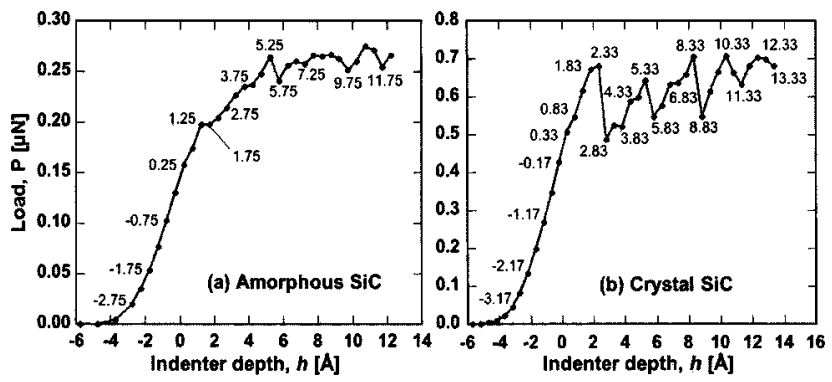

FIG. 1. Load-displacement response for (a) $a-\mathrm{SiC}$ and (b) 3C-SiC. 
forces). The load is calculated as the $z$ component of the force exerted on the indenter by the substrate averaged over the final $\sim 5 \mathrm{ps}$ of the holding phase.

The calculated load-displacement $(P-h)$ curve for $a$ $-\mathrm{SiC}$ is shown in Fig. 1(a). For comparison we plot the $P-h$ curve for a (110) surface of a crystalline $3 \mathrm{C}-\mathrm{SiC}$ system in Fig. 1(b). ${ }^{8}$ Both curves consist of a series of load drops. The major difference is that in the $3 \mathrm{C}-\mathrm{SiC}$, the drops occur at depths equally spaced by $\sim 3 \AA$, while in $a$-SiC they are irregularly spaced and take place at $h=1.75,4.25,5.75,7.25$, 9.75, and $11.75 \AA$. This irregular spacing reflects the lack of long-range order in $a$-SiC.

Despite the apparent similarity, the $P-h$ responses in $a$ $-\mathrm{SiC}$ and $3 \mathrm{C}-\mathrm{SiC}$ are quantitatively very different. The maximum indentation pressure (at the maximum load of $P_{\max }$ $\approx 0.27 \mu \mathrm{N}$ ) is only $40 \%$ of the corresponding value in $3 \mathrm{C}-$ $\mathrm{SiC}$. The hardness estimated from $P_{\max }$ divided by the indenter area $\sim 30 \mathrm{GPa}$ is in good agreement with experimental values. ${ }^{10}$ The magnitudes of load drops in $a-\mathrm{SiC}$ are smaller than in $3 \mathrm{C}-\mathrm{SiC}$. The first load drop in $a-\mathrm{SiC}$ occurs at a lower depth than in $3 \mathrm{C}-\mathrm{SiC}$, due to a lower intrinsic resistance to topological changes in amorphous materials. Through a series of unloading simulations, we have concluded that the onset of plastic deformation in $a$-SiC takes place at $h \approx 0.5 \AA$, in contrast to $h \approx 2 \AA$ in $3 \mathrm{C}-\mathrm{SiC}$. The difference is also reflected in the relaxation phase following each indentation step, which is on the average four times longer in $a$-SiC.

The periodic load drops in the $P-h$ response in $3 \mathrm{C}-\mathrm{SiC}$ have been shown to arise from the slipping of subsequent atomic layers in the substrate. ${ }^{8}$ Defects in amorphous materials are often described in terms of disclinations; ${ }^{16}$ however, it is not straightforward to analyze nanoindentation-induced deformation using this concept. Though there are no atomic layers in $a$-SiC, the analysis of the density profile along the $z$ direction in Fig. 2 reveals that the atomistic mechanism responsible for the load drops is similar to that of $3 \mathrm{C}-\mathrm{SiC}$. As shown in Fig. 2, there are considerable density oscillations close to the free surface. The first two maxima at $h=1.25$ and $3.75 \AA$ in the density of atoms, coincide with the first two load drops in the $P-h$ curve, analogously to the case of 3C-SiC. At larger depths, only small density oscillations prevail. Their wavelength $(\sim 2.5 \AA)$ is equal to the average dis

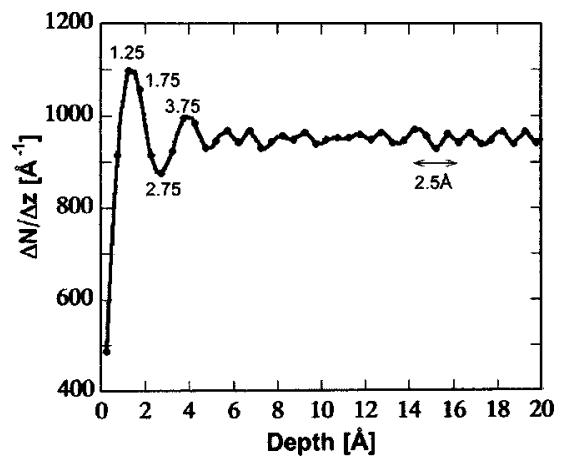

FIG. 2. Number of atoms in a $5 \AA$ horizontal slice vs its depth.

tance between atoms ( 3.08 and $1.98 \AA$ for homonuclear and heteronuclear bonds, respectively). This is because even in the absence of a crystallographic lattice, the short-range order is preserved in $a-\mathrm{SiC}$.

Structural defects are further analyzed by calculating bond-angle distribution in the material under the indenter. In the zinc-blende crystal, the $\mathrm{C}-\mathrm{Si}-\mathrm{C}$ and the $\mathrm{Si}-\mathrm{C}-\mathrm{Si}$ distribution exhibit a sharp peak at $\sim 109^{\circ}$ due to the tetrahedral arrangements of atoms. The distortion of these tetrahedral units during indentation results in the splitting of the single peak structure in the bond-angle distribution. This structure is restored after the load has dropped and the accumulated pressure has been released. ${ }^{8} \mathrm{In} a$-SiC, the peak in the $\mathrm{C}-\mathrm{Si}-\mathrm{C}$ bond-angle distribution becomes slightly narrower and higher during the load drop, reflecting the release of strain. However, the relaxation is much less pronounced than in $3 \mathrm{C}-\mathrm{SiC}$.

We also calculate the distribution of the local shear stress. In $a-\mathrm{SiC}$, it is evenly distributed in the entire substrate, taking values between -3 and $3 \mathrm{GPa}$, and no accumulation of shear stress is observed under the indenter. This is in contrast to $3 \mathrm{C}-\mathrm{SiC}$, in which the region of high shear stress clearly marks dislocations propagating in the substrate. ${ }^{8}$ The maximum local stress reached in $a$-SiC is one-sixth of that in $3 \mathrm{C}-\mathrm{SiC}$. The local pressure brings more information about deformations in $a$-SiC, as shown in Fig. 3(a) for $h=1.25 \AA$. The material beneath the indenter is characterized by regions of higher pressure (dark blue color), similarly to the case of 3C-SiC at $h=2.33 \AA$ [Fig. 3(b)]. In $a$-SiC, however, this effect is much less pronounced and
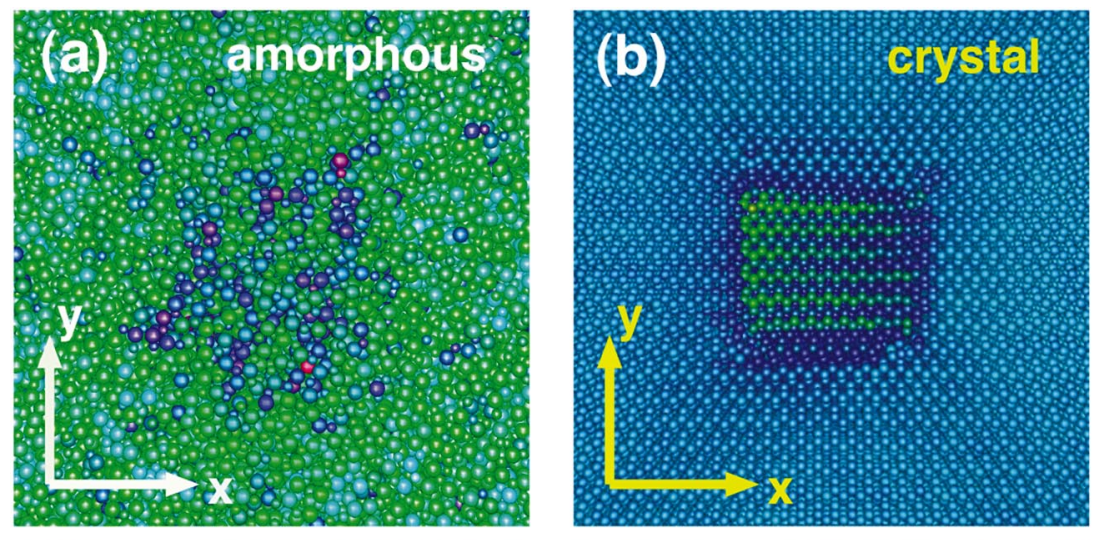

FIG. 3. (Color) Top view of the local pressure distribution in (a) $a-\mathrm{SiC}$ at $h$ $=1.25 \AA$ and (b) 3C-SiC at $h$ $=2.33 \AA$. 

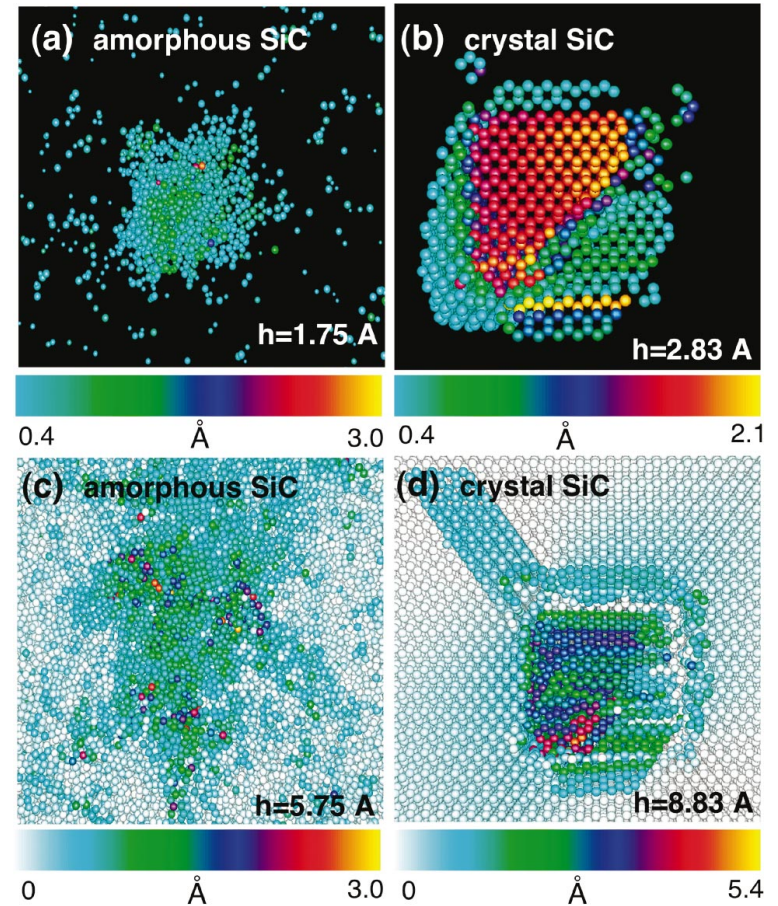

0

$\AA$

3.00

$\AA$

5.4

FIG. 4. (Color) Top views of the indented surfaces: (a),(c) $a$-SiC at $h$ $=1.75$ and $5.75 \AA$; (b),(d) $3 \mathrm{C}-\mathrm{SiC}$ at $h=2.83$ and $8.83 \AA$. Atoms are color coded by the displacement parameter $\Omega$.

pressure is much more irregularly distributed than in $3 \mathrm{C}-$ $\mathrm{SiC}$.

To specify the local rearrangements of atoms, we have introduced a displacement parameter defined as $\Omega=\mid \vec{r}_{n}(h)$ $-\vec{r}_{n}(h-0.5 \AA) \mid$, where $\vec{r}_{n}(h)$ is the position of the $n$th atom at the indenter depth $h$. We define perturbed atoms as those with $0.4 \AA \leqslant \Omega \leqslant 3 \AA$ and visualize them in Figs. 4(a) and 4(b) for $a-\mathrm{SiC}$ and $3 \mathrm{C}-\mathrm{SiC}$, respectively, after the occurrence of the first load drop in the corresponding $P-h$ responses. The shape of both distributions resembles the square base of the indenter, and the perturbed atoms are largely localized in the indentation region. In case of 3C-SiC [Fig. 4(b)], the diagonal line separating atoms with small (blue/green) and large (orange/yellow) values of $\Omega$ marks atoms sliding on the (111) glide plane of the zinc-blende structure of $3 \mathrm{C}-\mathrm{SiC}$ as discussed in Ref. 8.

The accumulation of high values of $\Omega$ in the indented region takes place also at larger indenter depths, as shown in Figs. 4(c) and 4(d) for $a$-SiC and 3C-SiC, respectively. Here we visualize all atoms with $\Omega \leqslant 3 \AA$. The deformations exhibit preferred directions or propagation, which are marked by light-blue bands of atoms on the surface. In case of 3C$\mathrm{SiC}$, the band in the top, left corner corresponds to a dislocation line propagating along the $[\overline{1} 1 \overline{1}]$ direction and was previously identified by means of the shortest-path ring analysis. ${ }^{8}$ The major difference between the two substrates is that in $a$-SiC [Figs. 4(a) and 4(c)] there is a non-negligible number of perturbed atoms distributed throughout the entire sample, whereas in 3C-SiC [Figs. 4(b) and 4(d)], the perturbed atoms are highly localized.

In order to quantify the localization of deformation, Fig. 5(a) shows the number $N(d)$ of perturbed atoms in spherical, 5-A-thick shells as a function of the distance $d$ from the center of indent. Both curves, for $3 \mathrm{C}-\mathrm{SiC}$ at $h=2.83 \AA$ (solid line) and $a$-SiC at $h=1.75 \AA$ (dashed line), exhibit a large peak around 20-30 A due to deformation near the indenter.
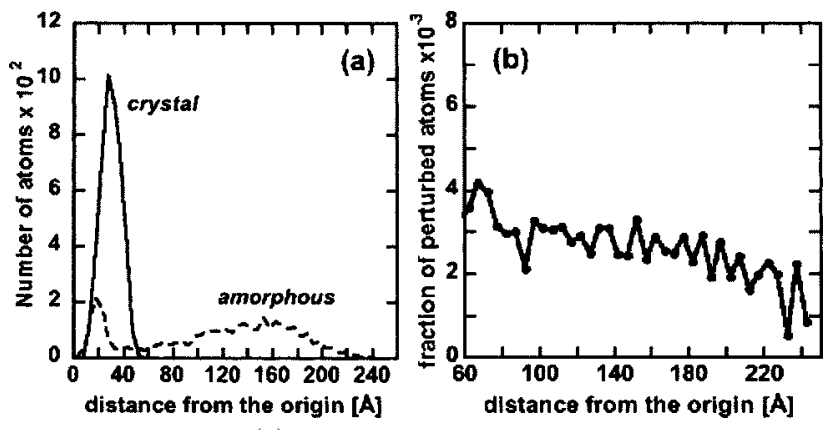

FIG. 5. (a) Number $N(d)$ of perturbed atoms as a function of distance $d$ from the center of indent for $a-\mathrm{SiC}$ (dashed line) and 3C-SiC (solid line). (b) The fraction of the density of perturbed atoms in $a$-SiC.

However, $a$-SiC also shows a long-range deformation extending to $d \sim 240 \AA$. The nature of this deformation becomes clear when we plot the density of perturbed atoms in $a$-SiC for $60 \AA \leqslant d \leqslant 250 \AA$ relative to the density of all atoms [see Fig. 5(b)]. The resulting curve shows characteristic oscillations, the wavelength of which coincides with half the indenter diagonal $(\sim 21 \AA)$.

In summary, we have performed MD simulations of nanoindentation of amorphous $\mathrm{SiC}$ and compared the results with a similar work done on crystalline $3 \mathrm{C}-\mathrm{SiC}$. The $P-h$ response of $a$-SiC exhibits a series of load drops, which are correlated with the changes in structural correlations. Magnitudes of load relaxed at the drops are smaller as compared with $3 \mathrm{C}-\mathrm{SiC}$, which is due to less accumulated pressure. The maximum indentation pressure is in good agreement with the experimental value of hardness for $a-\mathrm{SiC}(\sim 30 \mathrm{GPa})$ and is $60 \%$ lower than in $3 \mathrm{C}-\mathrm{SiC}$. The onset of plastic deformation occurs at a depth $\sim 25 \%$ of that in $3 \mathrm{C}-\mathrm{SiC}$. The indentation damage is more extended than in $3 \mathrm{C}-\mathrm{SiC}$, and it exhibits long-range oscillations consistent with the size of the indenter.

This work was supported by NSF, DOE, ARL-MURI, DARPA-PROM, AFOSR-DURINT, DOD Challenge, and CHSSI.

${ }^{1}$ J. Knap and M. Ortiz, Phys. Rev. Lett. 90, 226102 (2003).

${ }^{2}$ G. S. Smith, E. B. Tadmor, and E. Kaxiras, Phys. Rev. Lett. 84, 1260 (2000).

${ }^{3}$ J. Li, K. J. V. Vliet, T. Zhu, S. Yip, and S. Suresh, Nature (London) 418, 307 (2002).

${ }^{4}$ D. W. Brenner, S. B. Sinnott, J. A. Harrison, and O. A. Shenderova, Nanotechnology 7, 161 (1996).

${ }^{5}$ T. F. Page, W. C. Oliver, and C. J. McHargue, J. Mater. Res. 7, 450 (1992).

${ }^{6}$ K. J. V. Vliet, J. Li, T. Zhu, S. Yip, and S. Suresh, Phys. Rev. B 67, 104105 (2003).

${ }^{7}$ A. Gouldstone, K. J. V. Vliet, and S. Suresh, Nature (London) 411, 656 (2001).

${ }^{8}$ I. Szlufarska, R. K. Kalia, A. Nakano, and P. Vashishta, Appl. Phys. Lett. 85, 378 (2004).

${ }^{9}$ J. P. Rino, I. Ebbsjo, P. S. Braniccio, R. K. Kalia, A. Nakano, and P. Vashishta, Phys. Rev. B 70, 045207 (2004).

${ }^{10}$ M. A. E. Khakani, M. Chaker, A. Jean, S. Boily, J. C. Kieffer, M. E. O’Hern, M. F. Ravet, and F. Rousseaux, J. Mater. Res. 9, 96 (1994).

${ }^{11}$ W. R. Lambrecht, B. Segall, M. Methfessel, and M. van Schilfgaarde, Phys. Rev. B 44, 3685 (1991).

${ }^{12}$ F. Shimojo, I. Ebbsjo, R. K. Kalia, A. Nakano, and P. Vashishta, Phys. Rev. Lett. 84, 3338 (2000).

${ }^{13}$ M. Catti, Phys. Rev. Lett. 87, 033504 (2001).

${ }^{14}$ V. Heera, E. Prokert, N. Schell, H. Seifarth, W. Fukarek, M. Voelskow, and W. Skorupa., Appl. Phys. Lett. 70, 3531 (1997).

${ }^{15}$ V. B. Shenoy, R. Phillips, and E. B. Tadmor, J. Mech. Phys. Solids 48, 649 (2000).

${ }^{16}$ N. Rivier, Philos. Mag. A 40, 859 (1979). 